\title{
FUNCTIONS WHICH OPERATE IN THE FOURIER ALGEBRA OF A DISCRETE GROUP
}

\author{
LEONEDE de MICHELE AND PAOLO M. SOARDI ${ }^{1}$
}

ABSTRACT. In this paper we prove the following theorem: let $G$ be a discrete amenable group with nontrivial almost-periodic compactification, and let $F$ be a complex-valued function defined in $[-1,1]$; then $F$ operates in $A(G)$ if and only if $F$ is real-analytic in a neighborhood of the origin and $F(0)=0$.

1. Introduction. The Fourier algebra $A(G)$ of a locally compact group $G$ consists of those complex functions which are the convolution, $f * g$, of two functions $f, g \in L_{2}(G)$. The Fourier-Stieltjes algebra consists of those functions which can be written as linear combinations of continuous positive-definite functions in $G$ (we refer to $P$. Eymard [3] for definitions and notation). When $G$ is abelian with dual group $\Gamma$, the functions in $A(G)$ and $B(G)$ are the Fourier and Fourier-Stieltjes transforms of $L^{1}(\Gamma)$ and $M(\Gamma)$, respectively.

We will say that a complex function $F$, defined on a subset $E$ of the complex plane $C$, operates in $A(G)$ if the composition $F(f)$ belongs to $A(G)$ whenever $f \in A(G)$ and the range of $f$ is in $E$; a similar definition is given for $B(G)$. It is well known [5] that if $G$ is an infinite nondiscrete abelian group, and if $E$ is a closed convex set, then $F$ operates in $A(G)$ if and only if it is real-analytic on $E$; when $G$ is discrete abelian and $E$ an open set containing the origin, $F$ operates in $A(G)$ if and only if it is real-analytic in a neighborhood of zero and $F(0)=0$.

This result has been extended first by C. Dunkl [2] to compact groups containing an infinite abelian subgroup, and afterwards by D. Rider [8] to general infinite compact groups.

On account of a result of Herz (see for instance [4]), the restriction

Received by the editors September 13, 1973.

AMS (MOS) subject classifications (1970). Primary 43A13, 43A15.

Key words and phrases. Fourier algebra, locally compact groups, functions which operate, real-analytic.

1 The research of the second-named author was partially supported by C. N. R. within G. N. A. F. A. 
$\left.A(G)\right|_{H}$ of $A(G)$ to $H$ is exactly $A(H)$. Therefore, just as in [2], it is possible to extend the result of the abelian case to any locally compact group containing an infinite abelian subgroup. Notice that a nondiscrete Lie group satisfies this condition because it contains one-parameter subgroups. However, there are some groups which contain no infinite abelian subgroup.

In this paper we show that only real-analytic functions operate in $A(G)$ when $G$ is an infinite nonabelian discrete group with the following properties.

(1) $G$ is amenable, i.e. $A(G)$ contains a bounded approximate unit (see $[6])$.

(2) $G$ has nontrivial almost-periodic compactification; i.e., $G$ has infinitely many finite-dimensional irreducible representations.

The authors wish to thank Professor A. Figà-Talamanca for guidance and help in preparing this paper.

2. Statement and proof oi the Theorem. We prove the following

Theorem. Let $G$ be a discrete amenable group with nontrivial almostperiodic compactification, and let $F$ be a complex-valued function defined in $[-1,1]$. Then $F$ operates in $A(G)$ if and only if it is real-analytic in a neighborhood of zero and $F(0)=0$.

Remark. Obviously the same theorem holds if $F$ is defined in any complex neighborhood of zero.

To prove the Theorem, it suffices to establish some properties which are all that is needed in order to apply the proof of Helson-Kahane-KatznelsonRudin [5]. In the following, $F$ and $G$ will always satisfy the hypothesis stated in the Theorem; $\|\cdot\|$ will denote the norm in $B(G)$.

We start with

Proposition 1. There are $a \delta>0$ and $M>0$ such that $f \in B(G)$ and $\|f\|<\delta$ imply $F(f) \in B(G)$ and $\|F(f)\| \leq M$.

Proof. At first we prove the statement for finitely supported functions. If the proposition is false for these functions, then there exists a sequence $\left\{f_{n}\right\}$ with $\left\|f_{n}\right\| \leq 2^{-n}$ and $\left\|F\left(f_{n}\right)\right\| \rightarrow \infty, f_{n}$ finitely supported. Because of the amenability of $G$, we can find finitely supported functions $v_{n}$ such that $\left\|v_{n}\right\|<2$ and $v_{n}(x)=1$ on the support of $f_{n}$. We can also suppose that the sets $E_{n}=\operatorname{supp} v_{n}$ are mutually disjoint. Therefore,

$$
\sum_{n=1}^{\infty} f_{n}(x) \in A(G), \quad F\left(\sum_{n=1}^{\infty} f_{n}\right) \in A(G)
$$


and

$$
2\left\|F\left(\sum_{n=1}^{\infty} f_{n}\right)\right\| \geq\left\|v_{s} F\left(\sum_{n=1}^{\infty} f_{n}\right)\right\|=\left\|F\left(f_{s}\right)\right\|
$$

for every $s$, which is absurd.

Let now $f \in B(G)$ and $\|f\| \leq \delta / 2$; by the amenability of $G$, for every finite set $K \subset G$ there exists a finitely supported $v_{K} \in A(G)$ such that $\left\|v_{K}\right\| \leq$ 2 and $v_{K}(x)=1$ for every $x \in K$. Hence

$$
\left\|F\left(v_{K} f\right)\right\| \leq M \text { and } \lim _{K} F\left(v_{K} f\right)(x)=F(f)(x)
$$

for every $x \in G$. Therefore, by [3, Corollary 2.25], the proposition is proved.

Lemma. Let $G$ be an infinite discrete group. Then there exists a set $\Lambda \subseteq G$ with the following property: if $f \in B(G)$ and supp $f \subseteq \Lambda$, then $\operatorname{Inf}_{\mathbf{\Lambda}}|f(x)|=0$.

Proof. Assume, by way of contradiction, that the conclusion of the Lemma is false. Then for every $E \subseteq G$ there exists $f_{E} \in B(G)$ such that supp $f_{E}$ $\subseteq E$ and $\left|f_{E}(x)\right|>1$ for $x \in E$. Since $\bar{f}_{E} \in B(G)$, it follows that $f_{E} \bar{f}_{E}=\left|f_{E}\right|^{2}$ $\in B(G)$. Therefore, for some $M>0$,

$$
1<\left|f_{E}(x)\right|^{2}<M, \text { for all } x \in E \text {. }
$$

On the other hand (see [9, Theorem 13.9]), for every $\epsilon>0$ we can find a complex polynomial such that $|P(z)|<\epsilon$ in a neighborhood of the origin and $|P(z)-1|<\epsilon$ in a neighborhood of the interval $[1, M]$. Therefore, if $\chi_{E}$ denotes the characteristic function of $E,\left\|P\left(\left|f_{E}\right|^{2}\right)-\chi_{E}\right\|_{\infty}<\epsilon$. But $P\left(\left|f_{E}\right|^{2}\right) \epsilon$ $B(G)$, and hence we can approximate the characteristic function of every subset of $G$ with functions belonging to $B(G)$. By applying Theorem 3.3 of W. Bade and P. Cúrtis Jr. [1] to the Stone-ČCech compactification of $G$, we obtain $B(G)=L^{\infty}(G)$, which is absurd.

Remark. According to [7, Theorem 1], every discrete infinite group contains an infinite $\Lambda(4)$ set. On the other hand, every function in $B(G)$ which is supported on a $\Lambda(4)$ set is square summable, provided that $G$ is amenable. Thus for amenable groups a stronger version of the Lemma is true.

Proposition 2. $F$ is continuous in a neighborhood of zero.

Proof. We can always suppose $G$ countable; indeed, if $H$ is a countable subgroup of $G$ (for instance the subgroup generated by countably many distinct elements of $G), H$ is amenable and $A(H)=\left.A(G)\right|_{H^{*}}$.

Let $\Phi(s)=F\left(s_{0}+s\right)$, and let $\delta$ be the same number as in Proposition 1; 
we show that for every $\left|s_{0}\right|<\delta, \Phi(s)$ is continuous at zero. If not, then, since we can assume $\Phi(0)=0$, there would exist $\sigma>0$ and a sequence $\left\{a_{n}\right\}$ such that

$$
\sum_{n=1}^{\infty}\left|a_{n}\right| \leq \delta-\left|s_{0}\right| \text { and }\left|\Phi\left(a_{n}\right)\right|>\sigma .
$$

If $\Lambda=\left\{x_{k}\right\}$ is the same as in the Lemma, then the function $g=\sum_{k=1}^{\infty} a_{k} \delta_{x_{k}}$ belongs to $A(G)$ while $F(g) \notin B(G)$.

Proposition 3. Let $S_{r}$ be the set of real-valued $f \in B(G)$ with $\|f\|=r$. Then sup $\left\|e^{i f}\right\|=e^{r}, f \in S_{r}$.

Proof. Let $\bar{G}$ be the almost-periodic compactification of $G$; since $\bar{G}$ is infinite, the proposition is true for $\bar{G}$ by Rider [8, Lemma 1]. Therefore, by Eymard [3, Corollary 2.27], the proposition is true for $G$.

From Propositions 1, 2, and 3 and from Lemmas 2.3 and 2.4 in [5], the Theorem follows.

\section{REFERENCES}

1. W. G. Bade and P. C. Curtis Jro, Embedding theorems for commutative Banach algebras, Pacific J. Math. 18 (1966), 391-409. MR 34 \#1878.

2. C. F. Dunkl, Functions that operate in the Fourier algebra of a compact group, Proc. Amer. Math. Soc. 21 (1969), 540-544. MR 39 \#717.

3. P. Eymard, L'algèbre de Fourier d'un groupe localement compact, Bull. Soc. Math. France 92 (1964), 181-236. MR 37 \#208.

4. G. Flory, Eine Lebesgue-Zerlegung und functorielle Eigenschaften der Fourier Stieltjes Algebra, Doctoral Dissertation, University of Heidelberg, 1972.

5. H. Helson, J. P. Kahane, Y. Katznelson and W. Rudin, The functions which operate on Fourier transforms, Acta Math. 102 (1959), 135-157. MR 22 \#6980.

6. H. Leptin, Sur l'algèbre de Fourier d'un groupe localement compact, C. R. Acad. Sci. Paris Sér. A-B 266 (1968), A1180-A1182. MR 39 \# 362.

7. M. A. Picardello, Lacunary sets in discrete noncommutative groups, Boll. Un. Mat. Ital. (4) 8 (1973), 494-508.

8. D. Rider, Functions which operate in the Fourier algebra of a compact group, Proc. Amer. Math. Soc. 28 ( 197 1), 525-530. MR 43 \#2532.

9. W. Rudin, Real and complex analysis, McGraw-Hill, New York, 1966. MR 35 \#1420.

ISTITUTO MATEMATICO DELL' UNIVERSITA DI MILANO, VIA SALDINI 50, 20133 MILANO, ITALY 